\title{
ELM behaviour and linear MHD stability of edge ECRH heated ASDEX Upgrade plasmas
}

\author{
A. Burckhart, M. Dunne, E. Wolfrum, R. Fischer, R. \\ McDermott, E. Viezzer, M. Willensdorfer and the ASDEX \\ Upgrade Team \\ Max-Planck-Institut für Plasmaphysik, EURATOM Association, Garching, Germany \\ E-mail: andreas.burckhart@ipp.mpg.de
}

\begin{abstract}
In order to test the peeling-ballooning ELM model, ECRH heating was applied to the edge of ASDEX Upgrade type-I ELMy H-mode plasmas to alter the pedestal pressure and current density profiles. The discharges were analysed with respect to ideal MHD stability. While the ELM frequency increased and the pedestal gradients relaxed with edge ECRH, the MHD stability boundary did not change. The results indicate that the peeling-ballooning model is insufficient to fully explain the triggering of ELM instabilities in the presence of edge ECRH heating.
\end{abstract}

\section{Introduction}

Edge localised modes (ELMs) are magnetohydrodynamic (MHD) instabilities that occur at the edge of magnetically confined fusion plasmas. They periodically expel particles and energy from the confined region. In addition to limiting the confinement, they cause high heat fluxes to the walls of the tokamak which may not be manageable in larger, next-generation devices [1]. It will be necessary to control their amplitude and frequency in order to regulate these power loads and the impurity content of the plasma, as ELMs also expel impurities [2,3]. The most commonly invoked theory to explain the occurrence of ELMs is the peeling-ballooning model which posits a critical edge pressure gradient and current density [4].

Below these critical values, ELMs can prematurely be triggered with a local high density perturbation by injecting frozen deuterium pellets [5,6]. Experiments carried out at ASDEX Upgrade (AUG) showed that it is possible to pace ELMs by applying modulated electron cyclotron resonance heating (ECRH) power to the plasma edge [7]. However, this was only possible if the modulation frequency was close to the natural ELM frequency. More recently, it was found at the TCV tokamak that depositing continuous wave ECRH power at the edge of a plasma can increase the natural ELM frequency, while lowering the energy expelled per ELM [8]. However, a detailed analysis of the of kinetic profiles was not possible due to technical limitations of the experimental setup. 
The goal of the experiments presented in this publication is to test the peelingballooning theory by using edge ECRH to alter the edge pressure and current density profiles and investigate their effect on the ELM behaviour. A scenario was designed for AUG discharges in which the deposition location of ECRH heating was gradually shifted from the core to the edge. The kinetic edge profiles were compared in different phases and an ideal magnetohydrodynamic (MHD) stability analysis was performed. Furthermore, the possible role of electron cyclotron current drive (ECCD) in the different ELM behaviour was investigated.

\section{Experiments}

To analyse the MHD stability of the experiments, accurate magnetic equilibrium reconstructions of the plasma, particularly at the edge, are crucial. The magnetic reconstructions presented in this work were calculated using the interpretative equilibrium solver CLISTE [9], which is a further development of the Garching equilibrium code [10]. An equilibrium reconstruction requires, at a minimum, magnetic measurements of the plasma. At AUG, these measurements are provided by flux loops and poloidal and radial magnetic probes. In equilibria of elongated plasmas, reconstructed from magnetic data only, the total normalised pressure $\beta$ and current $I_{p}$ are very accurate. The current contained within the outermost percent of the confined plasma is also well known from the calculations [11]. However, the strong edge variation of the pressure gradient and current density peak are only poorly reflected. Constraining the edge pressure profile leads to a much higher accuracy in the final equilibrium [12]. This requires high quality kinetic measurements, which at AUG are provided by electron cyclotron emission (ECE) [13], Thomson scattering [14,15], lithiumbeam emission spectroscopy [16,17], deuterium cyanide interferometry [18] and charge exchange recombination spectroscopy [19] diagnostics.

\subsection{Edge ECRH heating}

All discharges were carried out at $800 \mathrm{kA},-2.5 \mathrm{~T}, 2.5 \mathrm{MW}$ neutral beam injection and a deuterium fuelling level of $4.5 \cdot 10^{21} \mathrm{~s}^{-1}$. Radial sweeps of the plasma were included to increase the spacial resolution of some diagnostics. Two discharges were performed to analyse the influence of edge ECRH heating on ELMs. In the reference discharge with 2.37 MW central ECRH the ELM frequency lay between 40 and $80 \mathrm{~Hz}$, but a few sporadic ELMs occurred already 6-8 ms after the previous event. Figure 1 shows that the discharge behaves like a typical type-I ELMy H-mode in AUG [20,21]. The ELM-induced energy losses are approximately $10 \%$, while the drop in edge density and in pedestal top temperature are slightly higher.

After gathering the reference data, a discharge was performed in which the ECRH power was gradually shifted toward the edge. However, a minimum amount of central ECRH heating was used at all times to prevent impurity accumulation. Figure 2 shows 


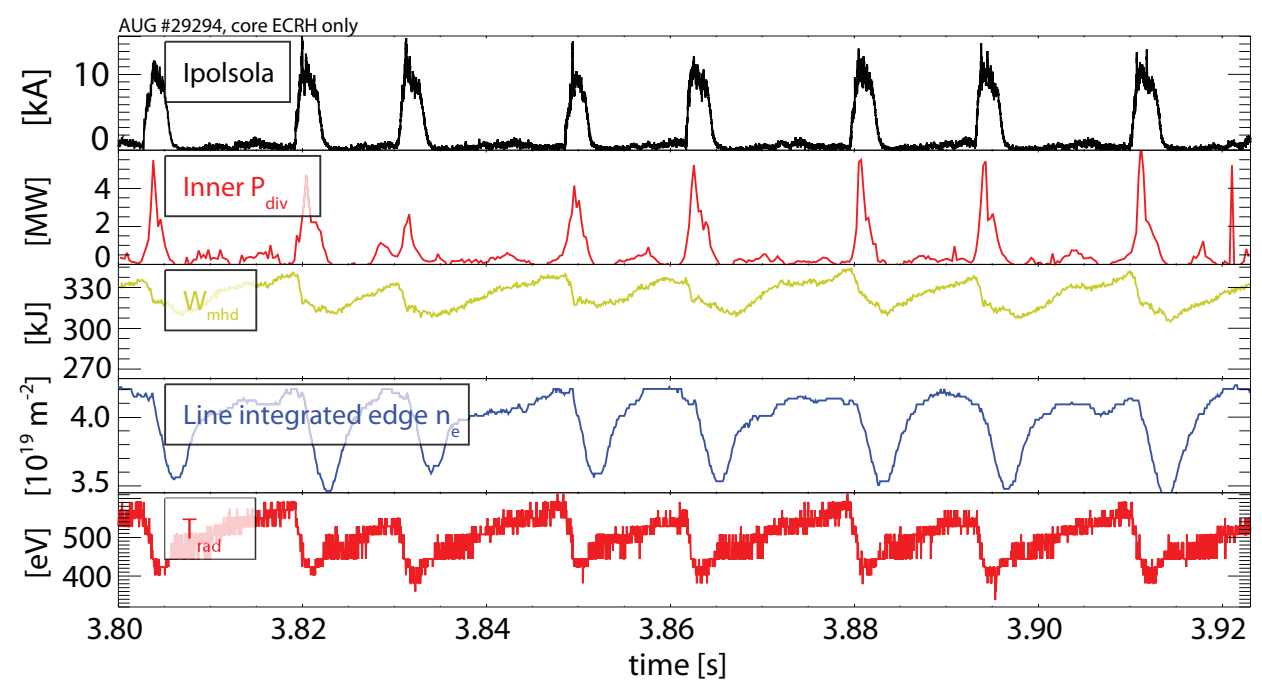

Figure 1. Type-I ELMs with central ECRH: time traces of the divertor current from shunt measurements (black), the power to the inner divertor (red), the total amount of energy confined in the plasma (green), the line integrated edge electron density (blue) and the ECE radiation temperature at the pedestal top (red)

the 2-D ECRH power deposition location (top) in the reference discharge and the three phases of the edge heated one, as well as the deposited power density as a function of the normalised radius (bottom). Note that while it appears that edge heating deposits less power, this is due to the power density being plotted per unit volume, which is much larger at the edge. The location and amplitude of the ECRH power deposited was calculated using the beam tracing code TORBEAM [22]. Before running the experiments, a test discharge was carried out with modulated ECRH power to verify the ECRH power deposition location as predicted by TORBEAM. The response of the ECE channels at the corresponding radius confirmed the predicted deposition location within $2 \mathrm{~cm}$, which was the resolution of the corresponding ECE channels in the test discharge.

In the discharges discussed here, the ELM frequency did not simply increase when shifting ECRH power to the edge, unlike observed at TCV [8]. Rather, the few sporadic ELMs already occurring 6-8 ms after the previous crash grew in number to form a second ELM frequency band between 100 and $130 \mathrm{~Hz}$ (see figure 3). As the ECRH heating was shifted toward the edge, this second band became more populated.

Figure 4 illustrates this trend. While the ELM frequency is still low when midradius heating is applied (left), it increases when the power from one gyrotron is shifted to the edge. However, one ELM cycle is longer, which results in the two frequency bands. In the last frame, which shows a time interval in which 1.6 MW of ECRH heating was deposited around $\rho_{p}=0.9$, only ELMs from the high frequency band can be seen. While the short time intervals shown in figure 4 were specifically chosen to illustrate the typical behaviour observed in this experiment, both frequency bands are still partly populated in the mid-radius and in the edge heating cases. However, the trend toward an increased 

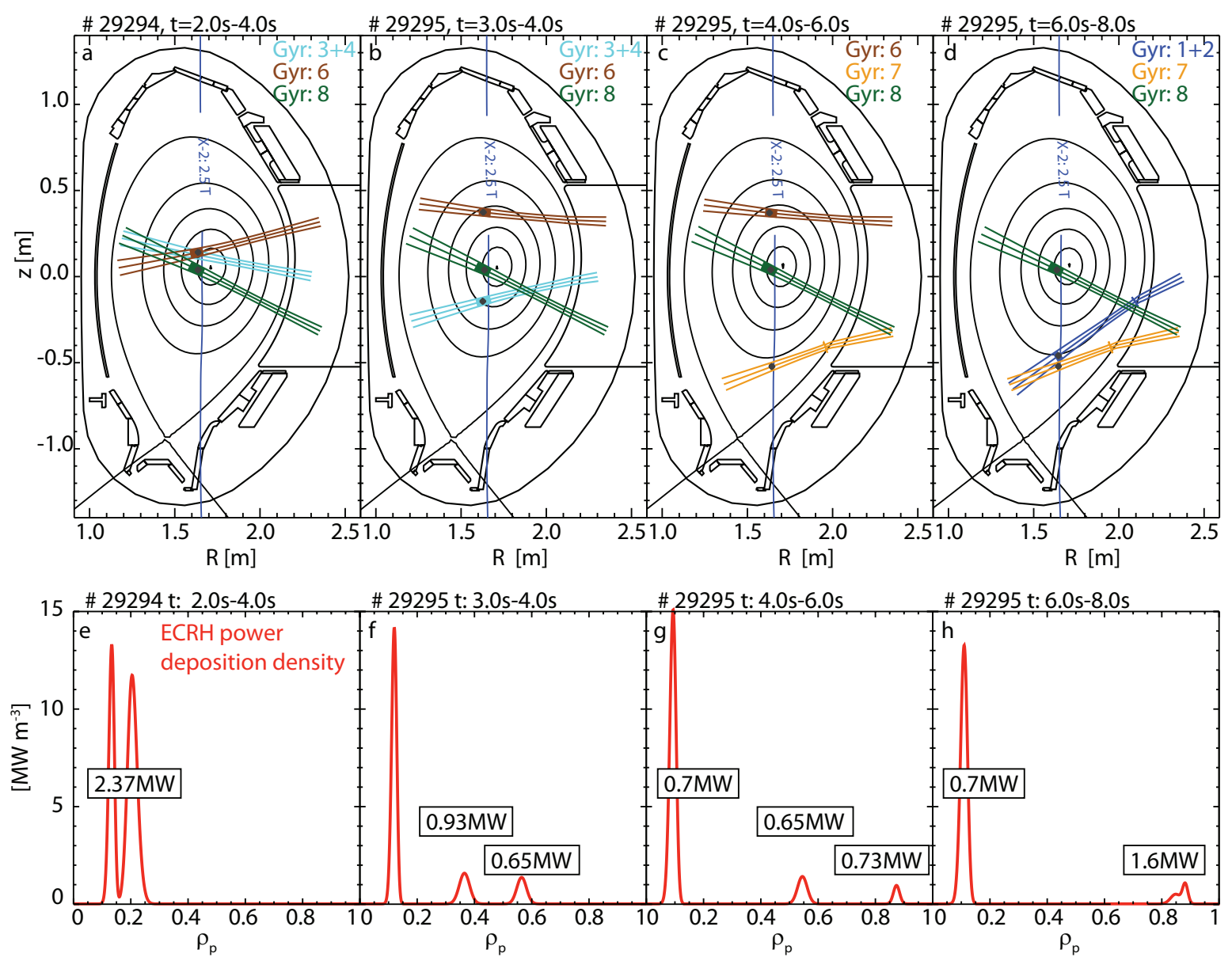

Figure 2. Poloidal cross section of $A U G$ showing the path and deposition location of the ECRH beams (top) and deposited power density (bottom) in the four considered phases

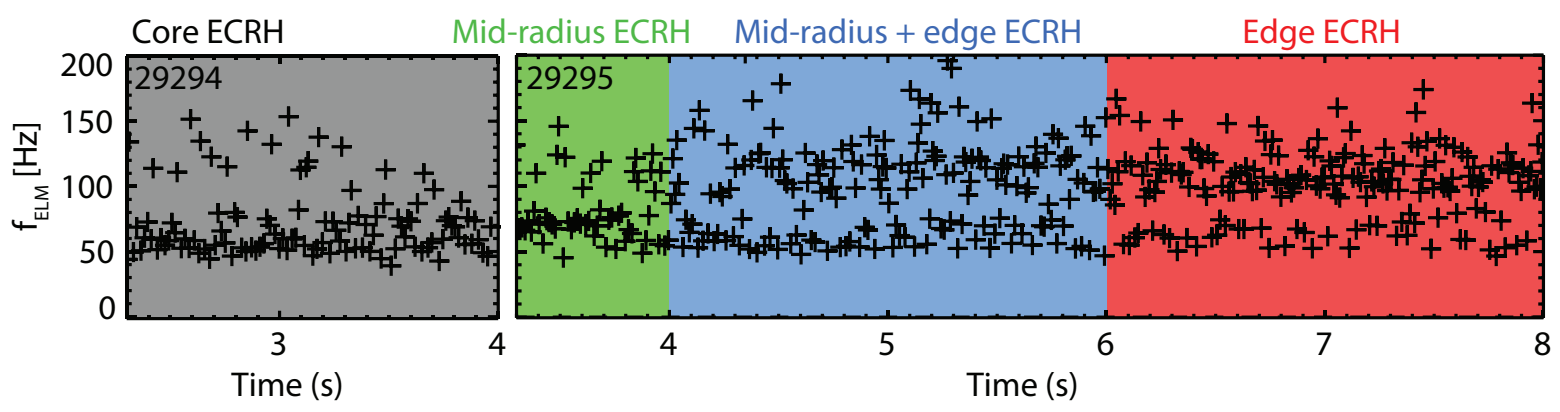

Figure 3. ELM frequency dependence of the ECRH power deposition location

population of the higher band with edge deposition is very clear (see figure 3). It can be seen in figure 5 that the ELMs from the upper frequency band induce roughly $50 \%$ less power loads to the divertor and cause half the energy loss. While the crash of the pedestal temperature is also roughly $50 \%$ lower, the particle losses are comparable. The $\mathrm{W}_{M H D}$ signals in figures 1 and 4 also show that the stored energy decreases as heating is shifted toward the edge. This is partly due to the core density becoming peaked in the 

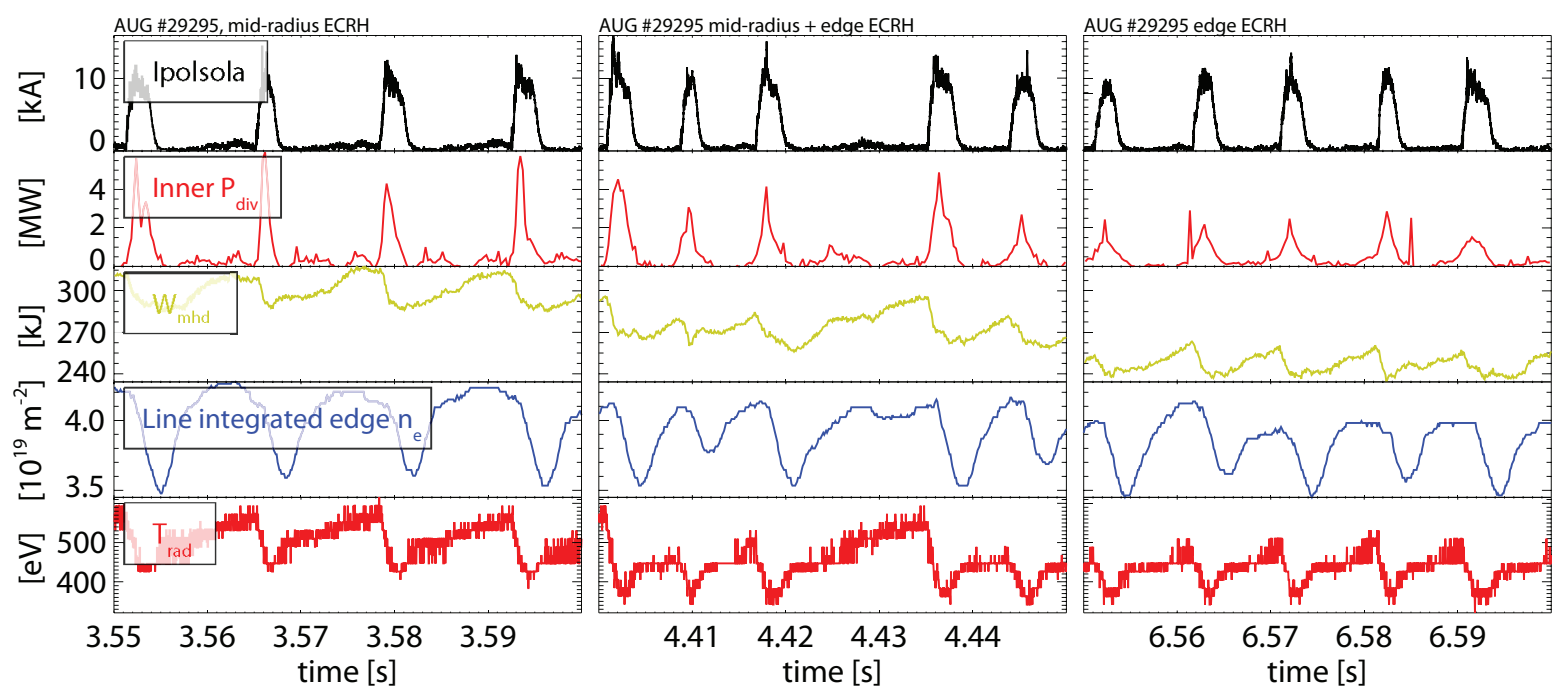

Figure 4. Type-I ELMs at different ECRH deposition locations: time traces of the divertor current from shunt measurements (black), the power to the inner divertor (red), the total amount of energy confined in the plasma (green), the line integrated edge electron density (blue) and the radiation temperature in the pedestal (red)
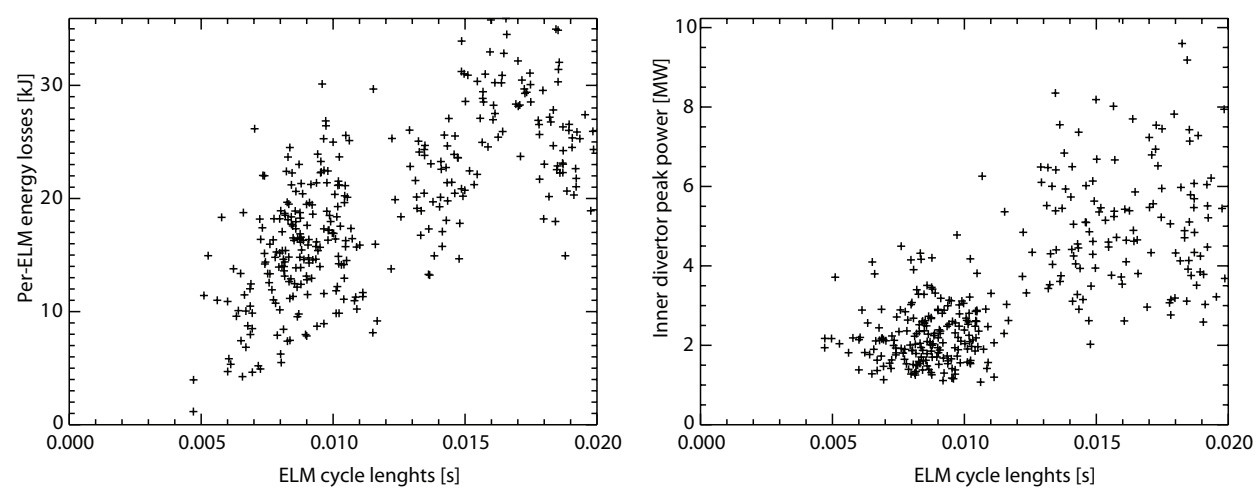

Figure 5. ELM energy losses (left) and power loads on the inner divertor (right)

presence of central ECRH and then flattening when part of the power is removed [23], and partly to a slightly lower mid-radius electron temperature. The density effect is also visible at the edge (see edge density traces in figure 4 left/right).

The kinetic profiles corresponding to three phases with different ECRH power deposition locations were ELM-synchronised between $0.1 \mathrm{~ms}$ and $3.5 \mathrm{~ms}$ before the ELM crash. Their fits are shown in figure 6. The pedestal top values of all kinetic profiles, $T_{e}, T_{i}$ and $n_{e}$, drop when shifting the ECRH deposition location to the edge. While the pressure gradient reaches $430 \mathrm{kPa} \mathrm{m}^{-1}$ in the core heated phase, it drops by $30 \%$ when moving the power deposition. The edge current density peak shows the same trend. With edge ECRH heating, ELMs are triggered faster than with core heating, even though the pressure gradient and current density are lower.

Since the reduction in $\mathrm{W}_{M H D}$ with edge ECRH heating is due both to reduced core heating and to lower density peaking, a similar experiment was performed in which the 

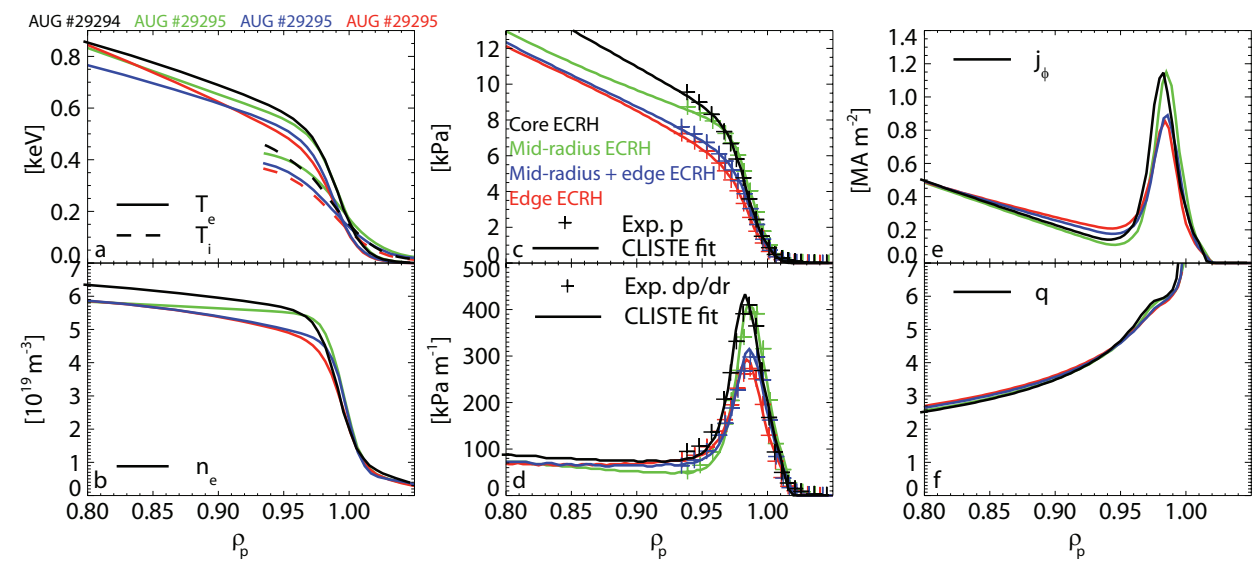

Figure 6. Pre-ELM profiles at different ECRH deposition locations of the electron and ion temperature (a), the electron density (b) the total pressure (c), its gradient (d), the toroidal current density at the LFS (e) and the q-profile (f)

core ECRH power was kept constant, but edge ECRH was added later in the discharge, thereby increasing the total heating power. The ELM frequency increased from 25$90 \mathrm{~Hz}$ initially to $70-200 \mathrm{~Hz}$ in the phase with additional edge ECRH. The pre-ELM $\mathrm{W}_{M H D}$ values dropped by $5 \%$ and the line integrated core density by $2 \%$ in the second phase, while no changes in the ELM losses or the edge density were observed. While the $T_{e}$ pedestal top value slightly increased with edge heating, $T_{i}$ decreased by $10 \%$. As the edge pressure gradient and current density both slightly dropped, the experiments suggest that these quantities cannot be responsible for the early ELM triggering.
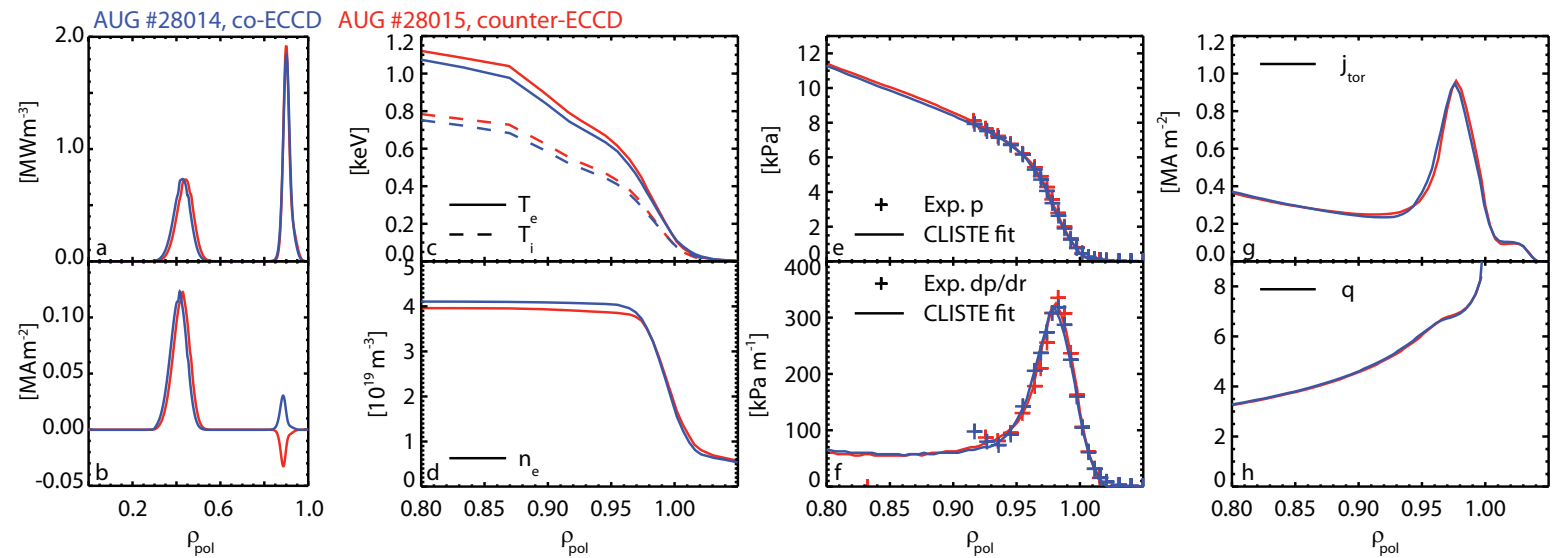

Figure 7. Pre-ELM profiles with co- (blue) and counter- (red) edge ECCD of the deposited power (a) and deposited current density (b), the electron and ion temperature (c), the electron density (d) the total pressure (e), its gradient ( $f)$, the toroidal current density at the $\operatorname{LFS}(g)$ and the q-profile $(h)$

In order to ensure that the changes in ELM behaviour do not result from additional edge current driven by ECCD [24], two discharges were carried out with opposite toroidal angles of the ECRH beam to drive current in the co- and the counter directions, thus yielding the maximum change in current between the two experiments. No change in 
the ELM frequency was observed between the two discharges.

The power and current deposition profiles can be seen in figure 7. Because of the low temperature at the plasma edge, TORBEAM calculations indicate that a total current of only $3.3 \mathrm{kA}$ was driven around $\rho_{p}=0.9$ by $3.1 \mathrm{MW}$ ECCD. All profiles, including the edge current density, are the same within the measurement errors and the CLISTE confidence bands. Both the experiment and the modelling suggest that an increase in the edge current due to ECCD cannot be the cause of the increased ELM frequency observed with edge ECRH deposition.

\section{Ideal MHD analysis}

In order to achieve the high degree of numerical accuracy required by stability codes, it is necessary to re-calculate the equilibrium by using a predictive equilibrium solver. In addition to the source profiles $p^{\prime}$ and $f f^{\prime}$, the fixed boundary code HELENA [25] requires the shape and location of the last closed flux surface. Both HELENA and the stability code ILSA [26] work in field aligned coordinates, which implies that they cannot resolve the separatrix at which the safety factor $\mathrm{q}$ and the magnetic shear reach infinity. Therefore, the X-point has to be rounded off [27] or the equilibrium is cut off at a certain radius. In all the results presented in this publication, the plasma was cut off at $99 \%$ of the poloidal flux at the separatrix.

Before running the actual stability code ILSA, the $\mathrm{j}-\alpha$ workflow, a subroutine of the Integrated Tokamak Modelling version of HELENA [26], is used to systematically alter the current density and pressure profiles independently. The pedestal top pressure and the edge current density peak are multiplied by different scaling factors in order to generate $j-\alpha$ diagrams which show the stability of the plasma versus the edge pressure gradient and current density as independent variables.

The peeling-ballooning stability of the analysed discharges was computed using ILSA, which is a framework built around the stability code CASTOR [26, 28-30]. In this work, ILSA was run in MISHKA-1 mode, which uses a reduced set of MHD equations [31]. It assumes an equilibrium velocity $\mathbf{v}_{0}=0$ and takes into account only a pressure perturbation instead of treating the density and temperature perturbations separately. The code calculates the growthrate $\gamma$ for different toroidal mode numbers n. A critical value for stability of $\gamma / \nu_{A}=0.04$ was chosen in all cases, with $\nu_{A}$ being the Alfvén frequency.

The diagrams in figure 8 illustrate the ideal linear stability of the four phases described earlier. The current density is averaged over the flux surface and the normalised pressure gradient is defined in reference [32] as

$$
\alpha=-\frac{2 V^{\prime}}{4 \pi^{2}} \sqrt{\frac{V}{2 \pi^{2} R_{0}}} \mu_{0} p^{\prime}
$$

where $\mathrm{V}$ is the plasma volume, $\mathrm{R}_{0}$ the major radius and $\mathrm{p}$ the total plasma pressure. The symbol ' represents the derivative with respect to the poloidal flux $\Psi$. The star in the figure represents the operational point, the values measured in the experimental plasma. 

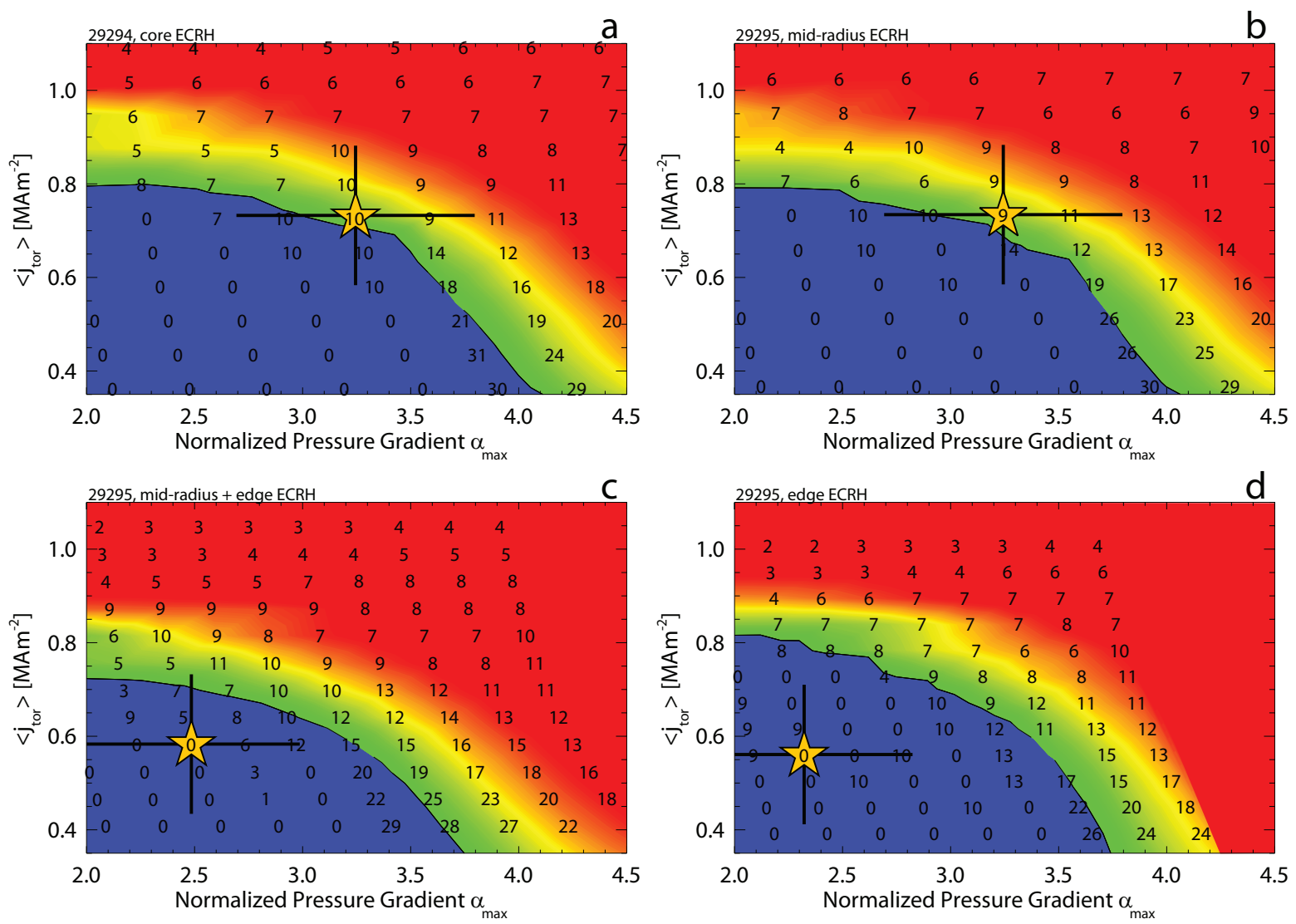

Figure 8. Stability diagrams of pre-ELM equilibria at different ECRH deposition locations

As was observed in the profiles in figure 6, the operational point moves toward lower pressure gradient and current density values as the heating power is shifted toward the edge. The stability boundary also moves slightly in the same direction due to a slightly broader pedestal and lower global $\beta$. However, this change of the stability boundary is much smaller than the shift of the operational point. While with core and mid-radius heating the operational point is at the marginally unstable region, the edge heated cases are stable. The decrease of the pressure gradient and the increasing population of the higher ELM frequency band with edge ECRH cannot directly be explained by ideal MHD.

As a shear of the plasma rotation has a stabilising effect on MHD modes [33], the earlier ELM onset could be explained by a reduction of the rotation at the edge. Such a trend is visible in the toroidal rotation data from the edge charge exchange recombination spectroscopy diagnostic as can be seen in figure 9 . In the mid-radius heating phase of discharge 29295 the rotation shear at the plasma edge is slightly stronger than in the edge heating phase. However, this trend is very close to the errors of the measurements and necessitates more detailed investigations. 


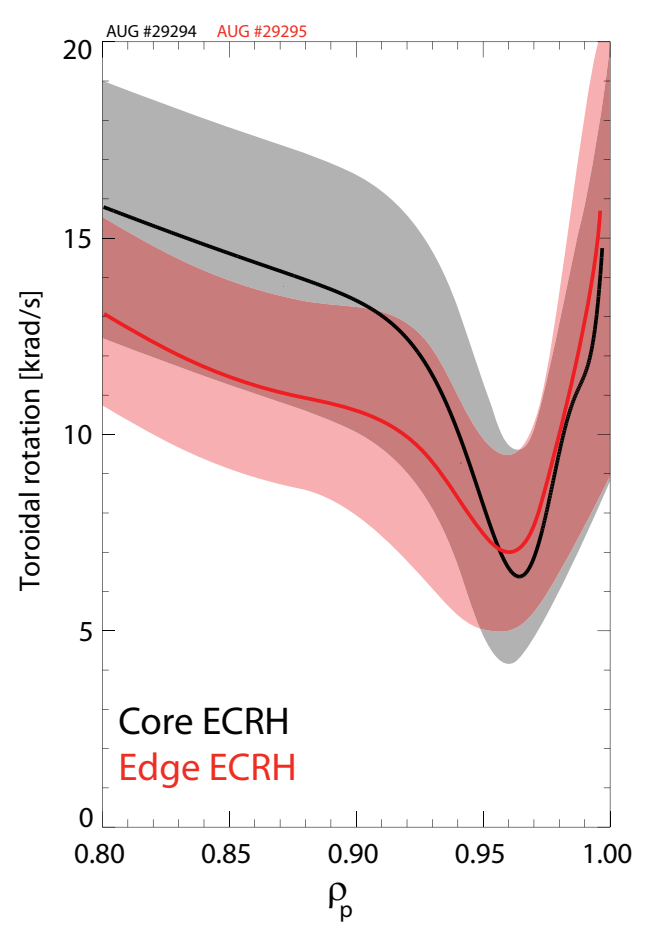

Figure 9. Toroidal rotation of impurity ions (boron) with core (black) and edge (red) ECRH heating

\section{Conclusion}

The influence of edge ECRH on the behaviour of ELMs was investigated at the ASDEX Upgrade tokamak. The ELM frequency did not increase linearly with the amount of heating power deposited at the edge, unlike in experiments performed at TCV. Rather, a second, higher ELM frequency band became more populated. However, the trend toward lower ELM energy losses with increasing ELM frequency was observed, but the total $\mathrm{W}_{M H D}$ decreased. From the point of view of peeling-ballooning theory, there is no reason that edge ECRH heating should lead to an earlier ELM crash. As the ECRH power deposition is shifted outwards, the operational point moves deeper into the stable region of the $j-\alpha$ diagram while the change of the stability boundary is much smaller. Dedicated discharges confirmed that no additional ECCD driven edge current is present when heating the plasma edge, and, therefore, can not explain the more unstable plasma edge. An observed decrease in the shear of the edge toroidal plasma rotation may be responsible for the unstable pedestal, but this trend is close to the measurements errors. Further dedicated experiments with focus on edge rotation are needed to investigate this in more detail.

\section{Acknowledgments}

This work has been carried out within the framework of the EUROfusion Consortium and has received funding from the Euratom research and training programme 2014- 
2018 under grant agreement No 633053. The views and opinions expressed herein do not necessarily reflect those of the European Commission.

\section{References}

[1] Federici G et al 2001 Nuclear Fusion 411967

[2] Zohm H 1996 Plasma Physics and Controlled Fusion 38 105-128

[3] Wade M R et al 2005 Physics of Plasmas 12056120

[4] Snyder P and Wilson H 2002 Contributions to Plasma Physics 42 258-271

[5] Baylor L, Jernigan T, Lasnier C, Maingi R, and Mahdavi M 1999 Journal of nuclear materials $266457-461$

[6] Lang P, Conway G, Eich T, Fattorini L, and Gruber O 2004 Nuclear fusion 44 665-677

[7] Horton L et al 2004 Plasma Physics and Controlled Fusion 46 B511

[8] Rossel J X 2012 Nuclear fusion 52032004

[9] McCarthy P 1999 Physics of Plasmas 6 3554-3560

[10] Lackner K 1976 Computer Physics Communications 12 33-44

[11] McCarthy P Jan. 2012 Plasma Physics and Controlled Fusion 54015010

[12] Dunne M et al 2012 Nuclear Fusion 52123014

[13] Hartfuss H, Geist T, and Hirsch M 1997 Plasma Physics and Controlled Fusion 39 1693-1769

[14] Murmann H, Gotsch S, Rohr H, Salzmann H, and Steuer K 1992 Review of scientific instruments 63 4941-4943

[15] Kurzan B and Murmann H D 2011 Review of scientific instruments 82103501

[16] Schweinzer J, Wolfrum E, Aumayr F, Pockl M, and Winter H 1992 Plasma Physics and Controlled Fusion 34 1173-1183

[17] Willensdorfer M et al 2012 Review of scientific instruments 83023501

[18] Mlynek A et al 2010 Review of scientific instruments 81033507

[19] Viezzer E, Pütterich T, Dux R, and McDermott R M 2012 Review of scientific instruments 83 103501

[20] Burckhart A, Wolfrum E, Fischer R, Lackner K, and Zohm H 2010 Plasma Physics and Controlled Fusion 52105010

[21] Schneider P A, Wolfrum E, Dunne M G, Dux R, and Gude A 2014 Plasma Physics and Controlled Fusion 56

[22] Poli E, Peeters A, and Pereverzev G 2001 Computer Physics Communications 13690 - 104

[23] Angioni C et al 2011 Nuclear Fusion 51023006

[24] Prater R 2004 Physics of Plasmas 112349

[25] Huysmans G, Goedbloed J, and Kerner W 1991 International Journal of Modern Physics C 2 $371-6$

[26] Konz C First physics applications of the Integrated Tokamak Modelling (ITM-TF) tools to the MHD stability analysis of experimental data and ITER scenarios in 38th EPS Conference on Plasma Physics, Strasbourg 2011, volume 35G, 2011

[27] Saarelma S, Kwon O J, and Kirk A 2011 Plasma Physics and Controlled Fusion 53

[28] Strumberger E, Gunter S, Merkel P, Riondato S, and Schwarz E 2005 Nuclear fusion 45 1156-1167

[29] Huysmans G, Goedbloed J, and Kerner W 1993 Physics of fluids. B, Plasma physics 5 1545-1558

[30] Kerner W, Goedbloed J, Huysmans G, Poedts S, and Schwarz E 1998 Journal of computational physics 142 271-303

[31] Mikhailovskii A and Sharapov S 1997 Plasma Physics Reports 23 844-57

[32] Miller R, Chu M, Greene J, Liu Y L, and Waltz R 1998 Physics of Plasmas 5 973-978

[33] Connor J W, Hastie R J, and Taylor J B 2004 Plasma Physics and Controlled Fusion 46 B1 